\title{
Dynamic Analysis and Driving Experiment of a Bicycle Focusing on Small Wheels*
}

\author{
Chihiro NAKAGAWA**, Shoichiro TAKEHARA*** and Yoshihiro SUDA**** \\ ${ }^{* *}$ Graduate School of Mechanical Engineering, Osaka Prefecture University \\ 1-1 Gakuen, Naka, Sakai, Osaka, Japan \\ E-mail: chihiro@iis.u-tokyo.ac.jp \\ ***Depatment of Mechanical Engineering, Tokyo Metropolitan University \\ 1-1, Minamiosawa, Hachioji, Tokyo, Japan \\ ****Institute of Industrial Science, University of Tokyo \\ 4-6-1, Komaba, Meguro, Tokyo, Japan
}

\begin{abstract}
In this research, the dynamic characteristics of bicycles are investigated focusing on small wheels. Multibody dynamics is used for the formulation of the bicycle model. The effects of the parameters of tire diameter and head angle are examined by focusing on a small wheel bicycle. The straight-ahead stability and upstanding stability are evaluated at each parameter. The results show the tendency of stability at each parameter and the influential parameter to the small wheel bicycle is found. The driving experiment using the small wheel bicycles with variable head angle is evaluated by the subjects. It is confirmed that varying head angle increases the stability of the small wheel bicycle. The results are corresponding with the simulation results and it is shown that the simulation captures the tendency of the stability and expresses the characteristic of the small wheel bicycle. Furthermore, the simulation using the effective parameters for small wheel bicycle was shown at the end. It showed the possibility of increase of the stability of a small wheel bicycle.
\end{abstract}

Key words: Transport, Simulation, Multibody Dynamics, Bicycle, Personal Mobility Vehicle

\section{Introduction}

A bicycle is a wide-spreading economical vehicle which has advantages in short trips, a parking space and health maintenance. It is a representative example of a personal mobility vehicle $[1,2]$ which is friendly for human and the environment. Especially in Japan, the heavy inbound traffic in metropolitan cities decreases the transportation efficiency and deteriorates the city environment by the air pollution and noise. It has been said that the spaces such as narrow roads, sidewalks and facilities need to be reconstructed in a practical and environmentally friendly manner for the sustainable development [3]. For such spaces, the compact personal mobility vehicles which have convenience and flexibility in the transportation are necessary [4]. Such a compact vehicle for individual usage has the potential to increase the convenience of the public transportation system [5]. For this back ground, a small wheel bicycle that is compact and easy to fold has attracted attention recently. There is the research ongoing which investigates the influence of the size and forms of the personal mobility vehicles on the pedestrians $[6,7]$ including the small wheel bicycle. When a small wheel bicycle is downsized, it increases the convenience; however, it 
decreases the stability and maneuverability at the same time. Therefore, it is necessary to investigate the stability and the maneuverability of the small wheel bicycle in order to develop a better personal mobility vehicle. The academic discussion about bicycles began from long ago $[8,9,10]$ but there have been few investigations on the dynamics of small wheel bicycles.

In our research, considering reducing tire diameter of a bicycle to develop a new personal mobility vehicle [11], the stability and maneuverability of a small wheel bicycle is investigated as the basic dynamic study.

First, we propose a three dimensional multibody bicycle model which can consider the nonlinearity of the system with parameter change. The use of the existing linear model and the commercial multibody software can be an option, however, the former is not necessarily appropriate when the dynamic analysis includes larger postural change and the latter has the black box nature. For the expansion of the model, such as adding the elastic system, control system design and linear system analysis [12], here the model is formed by using Newton-Euler equation.

Next, the relationship between the stability of bicycles and its wheel diameter is investigated by using the proposed model. The head angle which has influence on the stability of the bicycle is also discussed. The effect of the head angle on the small wheel bicycle is further examined by the driving experiments with several subjects.

\section{Small Wheel Bicycle}

Small wheel bicycles are defined as the bicycles its tire diameters are less than 20 inch $(0.5[\mathrm{~m}])$. Unlike the utility bicycles, they are not assumed to be used at high speed running (over $20[\mathrm{~km} / \mathrm{h}]$ ), many of them are designed in terms of portability and foldability[13]. Figure 1. shows the small wheel bicycle (tire diameter: 0.15 [m], mass: $12.2[\mathrm{~kg}]$ ) available in the market.

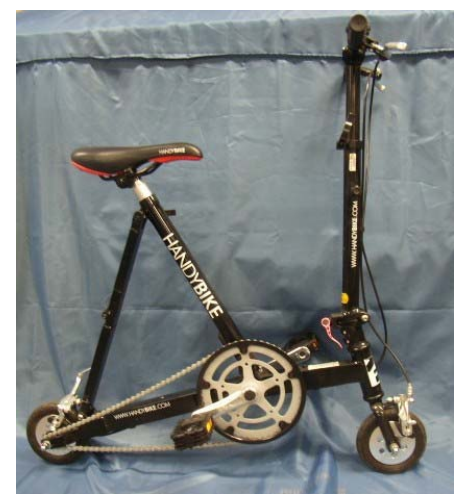

Fig. 1 Small wheel bicycle

\section{Modeling and Formulation}

\subsection{Modeling}

Figure 2 shows the bicycle model. The model consists of four rigid bodies; a front wheel, a rear wheel, a front frame and a rear frame fixing a rider. In this model, the wheels are circular disks without thickness; the bicycle moves on a flat road and wheels make point contact with the road without slip. Although using the constraint condition of point contact without slip is not necessarily appropriate to express the actual tire of the bicycle, in this paper, the formulation of the three dimensional motion is focused on. The four rigid bodies are constrained by three revolute joints at the front wheel axis, the rear wheel axis and the steer axis. The constraint condition with the road is calculated using position vector 


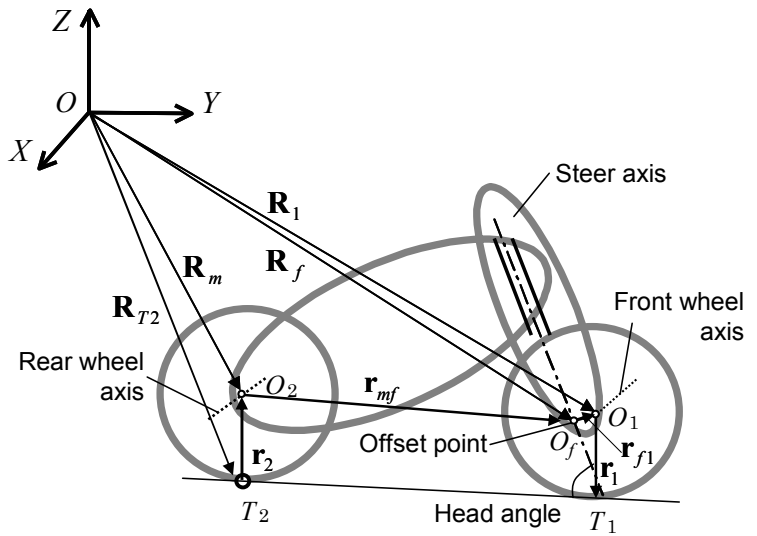

Fig. 2 Bicycle model

expressed a s $\mathbf{R}_{T 2}, \mathbf{R}_{m}, \mathbf{R}_{f}, \mathbf{R}_{1}$. These vectors are to the rear wheel contact point $\mathrm{T}_{2}$, the rear wheel center $\mathrm{O}_{2}$, the front wheel offset point $\mathrm{O}_{\mathrm{f}}$, the front wheel center from the inertial coordinate system $\mathrm{O}_{1}$, respectively. The offset is the distance between the steer axis and the front wheel center. The symbol $\mathrm{r}_{2}, \mathrm{rmf}_{\mathrm{mf}}, \mathrm{r}_{\mathrm{fl}}$ and $\mathrm{n}$ are the vector from the rear wheel contact point $T_{2}$ to the rear wheel center $\mathrm{O}_{2}$, from the rear center $\mathrm{O}_{2}$ to the front wheel offset point $\mathrm{O}_{\mathrm{f}}$, from the front wheel offset point $\mathrm{O}_{\mathrm{f}}$ to the front wheel center $\mathrm{O}_{1}$ and the front wheel center $\mathrm{O}_{1}$ to the front wheel contact point $T_{1}$, respectively. The inertial coordinate system has its origin on the road.

\subsection{Formulation}

The coordinate systems shown in Fig. 3 are defined as follows. The coordinate system $\Sigma^{O-X Y Z}$ is defined to be the inertial coordinate system, $\Sigma^{\mathrm{O}_{T 2}-\xi_{2} \eta_{2} Z} \mathrm{z}$ is the rear wheel contact point coordinate system, $\Sigma^{\mathrm{O}_{2}-\xi_{2} \eta_{2} \xi_{2}}$ is the rear wheel coordinate system, $\Sigma^{\mathrm{O}_{2}-\xi_{m} \eta_{m} \xi_{m}}$ is the rear frame coordinate system, and $\Sigma^{\mathrm{O}_{f}-\xi_{f} \eta_{f} \xi_{f}}$ is the front frame coordinate system. The vector $\mathbf{X}=\left(\begin{array}{lllllllll}X_{T 2} & Y_{T 2} & Z_{T 2} & \phi_{2} & \theta_{2} & \varphi_{m} & \phi_{s} & \varphi_{1} & \varphi_{2}\end{array}\right)^{T}$ is the generalized coordinate vector for the bicycle model. $\left(X_{T 2}, Y_{T 2}, Z_{T 2}\right)$ are the contact position of the rear wheel on the coordinate system $\Sigma^{\mathrm{O}_{T_{2}}-\xi_{2}^{\prime} \eta_{2} Z}$, the yaw angle $\phi_{2}$ is the rotation about $Z$-axis of $\Sigma^{\mathrm{O}_{T_{2}}-\xi_{2}^{\prime} \eta_{2} Z}$, the roll angle $\theta_{2}$ is the rotation about the $\xi_{2}$-axis of $\Sigma^{\mathrm{O}_{2}-\xi_{2} \eta_{2} \xi_{2}}$, the pitch angle $\varphi_{m}$ is the rotation about the $\zeta_{m}$-axis of $\Sigma^{\mathrm{O}_{2}-\xi_{m} \eta_{m} \zeta_{m}}$, and the steer angle $\phi_{f}$ is the rotation about the $\eta_{f}$-axis of $\Sigma^{\mathrm{O}_{f}-\xi_{f} \eta_{f} \xi_{f}}$. The equations of motion of the bicycle implicitly including the constraint at the front wheel axis, the rear wheel axis and the steer axis can be written as nine independent equation of motion by using the d'Alembert equation based on the virtual work principle. By the vector

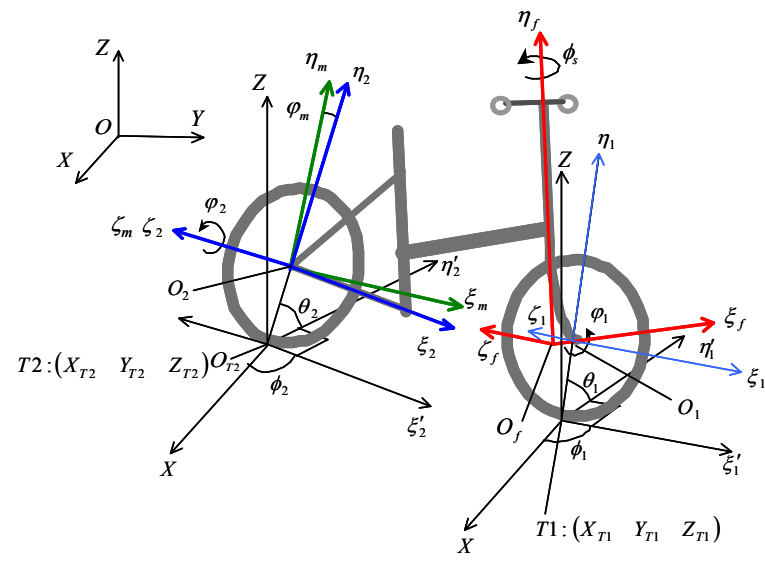

Fig. 3 Frame of reference of bicycle model 
notation, the equations of motion are handled without depending on the coordinate transformation. The tumbling condition and the contact condition are expressed by the Lagrange's method of undetermined multipliers. Using the differential algebraic equation has advantages in expanding the tumbling and contact conditions in detail.

The equations of motion are obtained as follows: Eq. 1 is the translational motion of the whole body; Eq. 2 is the yaw rotation of the whole body; Eq. 3 is the roll rotation of the whole body; Eq. 4 is the pitch rotation of the front wheel, the front frame and the rear frame about the axis of the rear wheel; Eq. 5 is the steering rotation of the front wheel and the front frame about the steering axis; Eq. 6 is the front wheel rotation about its axis; and Eq. 7 is the rear wheel rotation about its axis,

$$
\begin{aligned}
& \mathbf{F}_{2}+\mathbf{F}_{m}+\mathbf{F}_{f}+\mathbf{F}_{1}-\left(\dot{\mathbf{P}}_{2}+\dot{\mathbf{P}}_{m}+\dot{\mathbf{P}}_{f}+\dot{\mathbf{P}}_{1}\right) \\
& +\boldsymbol{\lambda}_{1}+\boldsymbol{\lambda}_{2}+\boldsymbol{\lambda}_{3}+\boldsymbol{\lambda}_{4}=\left.0\right|_{1,2,3} ^{\sum O-X Y Z} \\
& \sum_{i=1}^{N}\left(\mathbf{r}_{2}+\boldsymbol{\rho}_{2 i}\right) \times\left(\mathbf{F}_{2 i}-\dot{\mathbf{P}}_{2 i}\right) \\
& +\sum_{i=1}^{N}\left(\mathbf{r}_{2}+\boldsymbol{\rho}_{m i}\right) \times\left(\mathbf{F}_{m i}-\dot{\mathbf{P}}_{m i}\right) \\
& +\sum_{i=1}^{N}\left(\mathbf{r}_{2}+\mathbf{r}_{m f}+\boldsymbol{\rho}_{f i}\right) \times\left(\mathbf{F}_{f i}-\dot{\mathbf{P}}_{f i}\right) \\
& +\sum_{i=1}^{N}\left(\mathbf{r}_{2}+\mathbf{r}_{m f}+\mathbf{r}_{f i}+\boldsymbol{\rho}_{1 i}\right) \times\left(\mathbf{F}_{1 i}-\dot{\mathbf{P}}_{1 i}\right) \\
& +\left(\mathbf{r}_{2}+\mathbf{r}_{m f}+\mathbf{r}_{f 1}+\mathbf{r}_{1}\right) \times \lambda_{1} \\
& +\left(\mathbf{r}_{2}+\mathbf{r}_{m f}+\mathbf{r}_{f 1}+\mathbf{r}_{1}\right) \times \boldsymbol{\lambda}_{2}=\left.0\right|_{3} ^{\sum O_{T 2}-\xi_{2}^{\prime} \eta_{2}^{\prime} Z} \\
& \sum_{i=1}^{N}\left(\mathbf{r}_{2}+\boldsymbol{\rho}_{2 i}\right) \times\left(\mathbf{F}_{2 i}-\dot{\mathbf{P}}_{2 i}\right) \\
& +\sum_{i=1}^{N}\left(\mathbf{r}_{2}+\boldsymbol{\rho}_{m i}\right) \times\left(\mathbf{F}_{m i}-\dot{\mathbf{P}}_{m i}\right) \\
& +\sum_{i=1}^{N}\left(\mathbf{r}_{2}+\mathbf{r}_{m f}+\boldsymbol{\rho}_{f i}\right) \times\left(\mathbf{F}_{f i}-\dot{\mathbf{P}}_{f i}\right) \\
& +\sum_{i=1}^{N}\left(\mathbf{r}_{2}+\mathbf{r}_{m f}+\mathbf{r}_{f i}+\boldsymbol{\rho}_{1 i}\right) \times\left(\mathbf{F}_{1 i}-\dot{\mathbf{P}}_{1 i}\right) \\
& +\left(\mathbf{r}_{2}+\mathbf{r}_{m f}+\mathbf{r}_{f 1}+\mathbf{r}_{1}\right) \times \lambda_{1} \\
& +\left(\mathbf{r}_{2}+\mathbf{r}_{m f}+\mathbf{r}_{f 1}+\mathbf{r}_{1}\right) \times \boldsymbol{\lambda}_{2}=\left.0\right|_{1} ^{\sum O_{T 2}-\xi_{2} \eta_{2} \zeta_{2}} \\
& \sum_{i=1}^{N} \boldsymbol{\rho}_{m i} \times\left(\mathbf{F}_{m i}-\dot{\mathbf{P}}_{m i}\right) \\
& +\sum_{i=1}^{N}\left(\mathbf{r}_{m f}+\boldsymbol{\rho}_{f i}\right) \times\left(\mathbf{F}_{f i}-\dot{\mathbf{P}}_{f i}\right) \\
& +\sum_{i=1}^{N}\left(\mathbf{r}_{m f}+\mathbf{r}_{f 1}+\boldsymbol{\rho}_{1 i}\right) \times\left(\mathbf{F}_{1 i}-\dot{\mathbf{P}}_{1 i}\right) \\
& +\left(\mathbf{r}_{m f}+\mathbf{r}_{f 1}+\mathbf{r}_{1}\right) \times \lambda_{1} \\
& +\left(\mathbf{r}_{m f}+\mathbf{r}_{f 1}+\mathbf{r}_{1}\right) \times \lambda_{2}=\left.0\right|_{3} ^{\sum O_{2}-\xi_{m} \eta_{m} \zeta_{m}}
\end{aligned}
$$




$$
\begin{aligned}
& \sum_{i=1}^{N} \boldsymbol{\rho}_{f i} \times\left(\mathbf{F}_{f i}-\dot{\mathbf{P}}_{f i}\right) \\
&+ \sum_{i=1}^{N}\left(\mathbf{r}_{f 1}+\boldsymbol{\rho}_{1 i}\right) \times\left(\mathbf{F}_{1 i}-\dot{\mathbf{P}}_{1 i}\right) \\
&+\left(\mathbf{r}_{f 1}+\mathbf{r}_{1}\right) \times \boldsymbol{\lambda}_{1}+\left(\mathbf{r}_{f 1}+\mathbf{r}_{1}\right) \times \boldsymbol{\lambda}_{2} \\
&=\left.0\right|_{2} ^{\sum O_{f}-\xi_{f} \eta_{f} \xi_{f}} \\
& \sum_{i=1}^{N} \boldsymbol{\rho}_{1 i} \times\left(\mathbf{F}_{1 i}-\dot{\mathbf{P}}_{1 i}\right)+\mathbf{r}_{1} \times \boldsymbol{\lambda}_{1}=\left.0\right|_{3} ^{\sum o_{f}-\xi_{f} \eta_{f} \xi_{f}} \\
& \sum_{i=1}^{N} \boldsymbol{\rho}_{2 i} \times\left(\mathbf{F}_{2 i}-\dot{\mathbf{P}}_{2 i}\right)-\mathbf{r}_{2} \times \boldsymbol{\lambda}_{3}=\left.0\right|_{3} ^{\sum o_{2}-\xi_{2} \eta_{2} \zeta_{2}},
\end{aligned}
$$

where, $\mathbf{F}$ is external force applied to each body and $\mathbf{P}$ is the momentum of each body. The lower-right subscripts on $\mathbf{F}$ and $\mathbf{P}$ indicate the body number. Body number 1 indicates the front wheel, body number 2 indicates the rear wheel, body number $m$ indicates the rear frame, and body number $f$ indicates the front frame. The constraint forces $\lambda$ are numbered as follows: $\lambda_{1}$ is the resultant of the traction force and side force on the contact point of the front wheel, $\lambda_{2}$ is the reaction force at the front wheel contact point, $\lambda_{3}$ is the resultant of the traction force and side force on the contact point of the rear wheel, and $\lambda_{4}$ is the reaction force at the rear wheel contact point. $i$ is the subscript given to the point masses that compose each body; each body is assumed to consist of $N$ mass points. Making $N$ to approach infinity and summing the mass points of each body expresses the rigid body. The symbol $\rho_{x i}$ is the vector from coordinate system $x$ to point mass $i$. The upper-right subscript indicates the coordinate systems; the lower-right subscript indicates the element number in the equation.

The constraint equations are as follows: Eq. 8 and 9 represents the front wheel tumbling condition and contact condition; Eq. 10 and 11 represents the rear wheel tumbling condition and contact condition. The symbol $\omega_{2}$ is the angle rate of the rear wheel center coordinate, the symbol $\mathbf{n}_{Z}$ is the unit vector for $Z$ direction.

$$
\begin{aligned}
& \dot{\mathbf{R}}_{T 2}+\omega_{2} \times \mathbf{r}_{2}+\left(\omega_{2}+\dot{\varphi}_{m}\right) \times \mathbf{r}_{m f} \\
& +\left(\omega_{2}+\dot{\varphi}_{m}+\dot{\phi}_{S}\right) \times \mathbf{r}_{f 1} \\
& +\left(\omega_{2}+\dot{\varphi}_{m}+\dot{\boldsymbol{\phi}}_{S}+\dot{\varphi}_{1}\right) \times \mathbf{r}_{1}=\mathbf{0}||_{1,2}^{\sum_{T 1}-\xi_{1}^{\prime} \eta_{1}^{\prime} Z} \\
& \left(\mathbf{R}_{T 2}+\mathbf{r}_{2}+\mathbf{r}_{m f}+\mathbf{r}_{f 1}+\mathbf{r}_{1}\right) \cdot \mathbf{n}_{Z}=\mathbf{0}, \\
& \dot{\mathbf{R}}_{T 2}-\dot{\varphi}_{2} \times \mathbf{r}_{2}=\mathbf{0}||_{1,2}^{\sum O_{T 2}-\xi_{2}^{\prime} \eta_{2}^{\prime} Z} \\
& \mathbf{R}_{T 2} \cdot \mathbf{n}_{z}=\mathbf{0},
\end{aligned}
$$

where, the general form of obtained differential-algebraic equations (DAEs) can be written as follows:

$$
\left[\begin{array}{cc}
\mathbf{M} & \mathbf{C}_{\mathbf{q}}^{T} \\
\mathbf{C}_{\mathbf{q}} & \mathbf{0}
\end{array}\right]\left[\begin{array}{l}
\ddot{\mathbf{q}} \\
\boldsymbol{\lambda}
\end{array}\right]=\left[\begin{array}{l}
\mathbf{F} \\
\boldsymbol{\gamma}
\end{array}\right]
$$

where $\mathrm{M}$ is the mass matrix, $\mathbf{C}_{\mathbf{q}}$ is the Jacobian matrix, $\boldsymbol{\lambda}$ are the Lagrange multipliers, $\mathbf{F}$ are the external forces, and $\gamma$ are acceleration equations.

\section{Numerical Simulation}

In this chapter, using the proposed model, the stability of the bicycle is discussed focusing on the parameters of wheel diameter. 


\subsection{Simulation Condition}

To investigate the stability of the bicycle itself, the riding without steering control is discussed. The bicycle begins the experiment moving in a straight line with an initial disturbance of $0.26 \mathrm{~N} \cdot \mathrm{m}$ to the steer angle. The front and rear frames are assumed to be homogeneous rectangular solids (length*height*width: $0.035^{*} 0.8 * 0.2[\mathrm{~m}], 0.6 * 1.0 * 0.25$ [m], respectively) and each inertial moment around the central axis is $0.18,0.01,0.17$ $\left[\mathrm{kgm}^{2}\right], 6.99,2.78,8.95\left[\mathrm{kgm}^{2}\right]$, respectively. The wheels are assumed to be circular hollow disks with $0.1[\mathrm{~m}]$ distance between the outside diameter and inside diameter. The steering axis and the axis of inertia in the vertical direction are assumed to be parallel. The damping force for the rotation around the front and rear wheel axis and steer axis, the rolling resistance at the contact area, the air resistance and the driving torque added to the rear wheel axis are ignored. Table 1 shows the parameters of the 6- and 24-inch bicycles. Here we define the 6-inch bicycle as a small wheel bicycle and the 24-inch bicycle as a utility bicycle. The head angle is the angle between horizontal line and the steer axis. It is synonymous for the complementary angle of the caster angle that is the angle between the vertical axis and the steer axis. The Runge-Kutta method is used in the numerical integration by $0.01[\mathrm{~s}]$.

Table 1 Parameters of Bicycle

\begin{tabular}{lll}
\hline Description & 6 inch bicycle & 24 inch bicycle \\
\hline Mass of main frame & $79 \mathrm{~kg}$ & $79 \mathrm{~kg}$ \\
Mass of front frame & $3.2 \mathrm{~kg}$ & $3.2 \mathrm{~kg}$ \\
Mass of tire & $0.62 \mathrm{~kg}$ & $2.2 \mathrm{~kg}$ \\
Wheel diameter & $0.15 \mathrm{~m}$ & $0.60 \mathrm{~m}$ \\
Wheelbase & $0.75 \mathrm{~m}$ & $0.913 \mathrm{~m}$ \\
Offset & $0.035 \mathrm{~m}$ & $0.035 \mathrm{~m}$ \\
Head angle & $72.5 \mathrm{deg}$ & $72.5 \mathrm{deg}$ \\
\hline
\end{tabular}

\subsection{Stability Criteria}

The stability criteria are determined according to the Japan Industrial Standards Bicycle Drivability Test method [14] and the experiments found in [15]. The stability time is defined as the time till the lateral translation of the bicycle reaches $\pm 0.25 \mathrm{~m}$ from the centerline and the roll angle reaches \pm 20 degrees from the upright position. Figure 4, 5 show the schematic view of the stability criteria of bicycles.

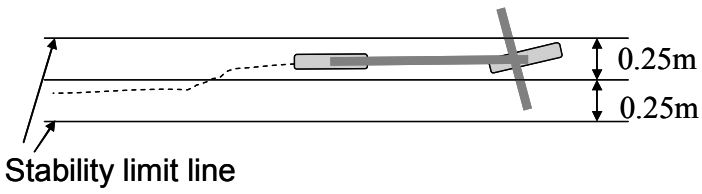

Fig. 4 Stability limit for lateral translation

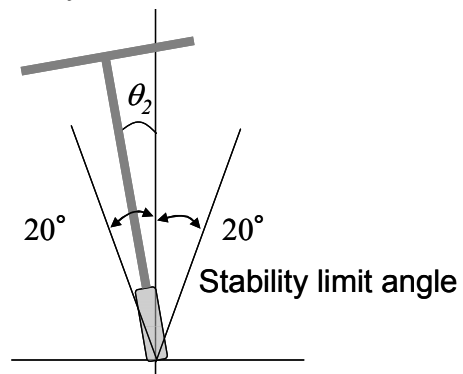

Fig. 5 Stability limit for roll angle 


\subsection{Comparison between the Small Wheel Bicycle and the Utility Bicycle}

To investigate the stability of the small wheel bicycle, the simulation results of the small wheel bicycle and the utility bicycle are compared. Figure 6 shows the trajectories of the 6- and 24-inch bicycles at a velocity of $2.5 \mathrm{~m} / \mathrm{s}$. The horizontal axis is the direction the bicycles run and the vertical axis is the lateral translation of the bicycles. It is clear that the 6-inch bicycle is less stable than the 24 -inch bicycle by $80 \%$ of its stabilizing distance. The stability time has also the same tendency. When the disturbance to the steer angle becomes larger, the trajectories of the 6- and 24-inch bicycles become shorter for the direction the bicycles run and vice versa. At the realistic range of the disturbance that the bicycle can run, the trajectory of the bicycle oscillates from side to side qualitatively. From dynamic point of view, the trajectory of the bicycle is depicted to the direction of the steering disturbance and goes back to the reference line by the trail effect etc. At this time, the roll angle of the bicycle tries to back to the upright position by the effect. The trajectories are depicted by this repetition. In case of 6-inch bicycle, the trail effect and gyro effect are less than that of 24-inch bicycle, then the trajectory of the 6-inch bicycle get to the lateral limit and roll angle limit faster. From this, it is found that the stability of the small wheel bicycle is quite inferior to that of the utility bicycle.

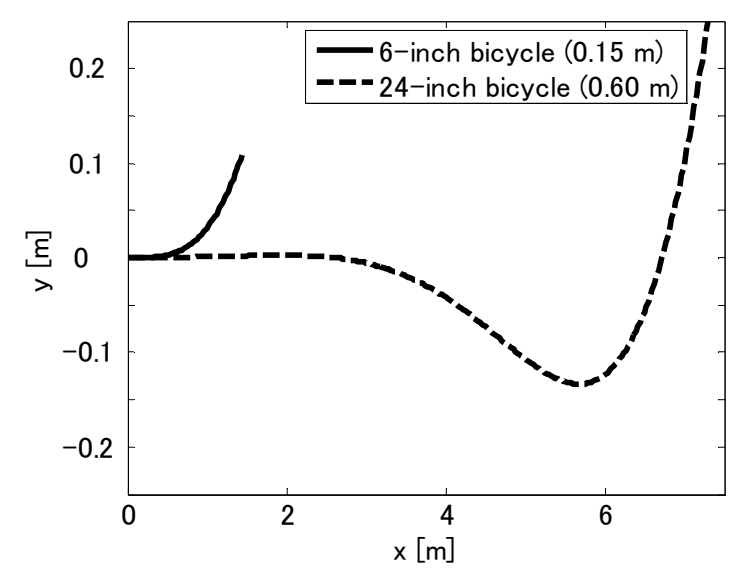

Fig. 6 Trajectories of bicycles

\subsection{Influence of Wheel Diameter}

Next, the influence of the small wheel diameter on the stability of the bicycle is investigated. Here, we define the wheels as circular hollow disks with $0.1[\mathrm{~m}]$ distance between the outside diameter and inside diameter. The inertia moment of the wheel becomes different according to the wheel diameter. This premise considering the influence of the wheel inertia moment is in practical steps. The influence of the inertia moment is further discussed at section 4.5 in detail. Figure 7 shows the stability time of the lateral translation and roll angle. The wheel diameter is varied from $0.1 \mathrm{~m}$ to $0.65 \mathrm{~m}$. The horizontal axis shows the wheel diameter and the vertical axis shows the stability time defined in the section 4.2 . The velocity of the bicycle in the city is said to be at 2 to $4[\mathrm{~m} / \mathrm{s}]$ [16] . Figure 7 shows the case with the initial velocity 2 and $4[\mathrm{~m} / \mathrm{s}]$. In case of the initial velocity $4[\mathrm{~m} / \mathrm{s}]$, the roll angle is within -20 to 20 degree over the wheel diameter $0.25[\mathrm{~m}]$, therefore the stability time for roll angle is not plotted. The wheel diameter $0.15[\mathrm{~m}]$ bicycle is the 6 inch bicycle and $0.6[\mathrm{~m}]$ is the 24 inch bicycle. From the results, it is shown that bicycles with larger wheel diameters are more stable, which phenomenon agrees with the empirical recognition. The variation of the stability time for the lateral translation and the roll angle as the wheel diameter is changed are almost the same. Thus, it is proved to be useful to adopt above stability criteria. From this, here, the stability time is defined as the 
time that bicycles reaches either stability limit. Figure 7 also shows that the decreasing rate of the stability time for wheel diameters under $0.25 \mathrm{~m}$ is large compared to that of a wheel diameters over $0.25 \mathrm{~m}$. This indicates that wheel diameter has a strong influence on the bicycle dynamics, especially for small wheel bicycles.

\subsection{Influence of Head Angle}

The head angle, which is an influential parameter in the stability of two-wheeled vehicles, is discussed. Generally, the head angle of bicycles is set from 66 to 75 degree [17]. Considering the range, the stabilities of the bicycles with the head angle 70, 72.5 and 75 degree are investigated as their wheel diameters vary. In the simulation, the velocity of the bicycles is $2.5[\mathrm{~m} / \mathrm{s}]$. Figure 8 shows that, in the range of small wheel bicycle from $0.1 \mathrm{~m}$ to $0.25 \mathrm{~m}$ wheel diameter, smaller head angles have more stability. The trail effect may be the reason for this [18]. Especially, the small wheel bicycle has smaller wheel diameter and

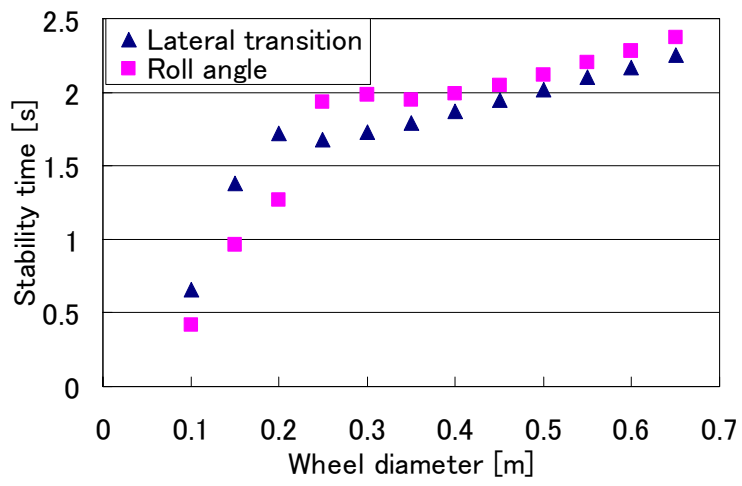

(a) $2[\mathrm{~m} / \mathrm{s}]$

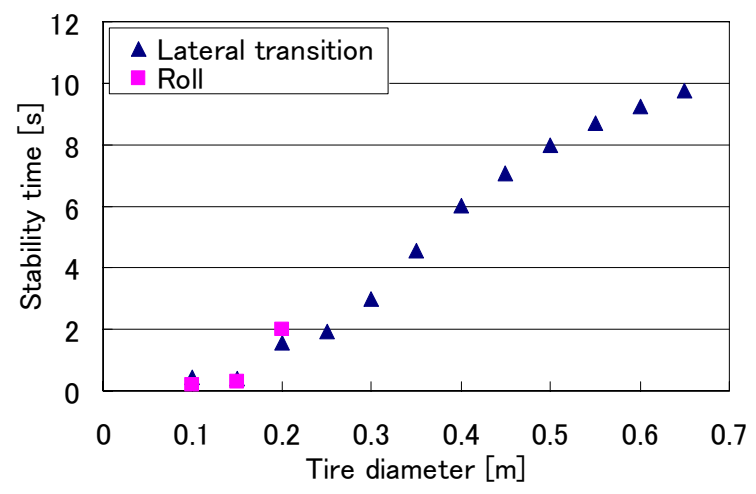

(b) $4[\mathrm{~m} / \mathrm{s}]$

Fig. 7 Stability in different tire diameter

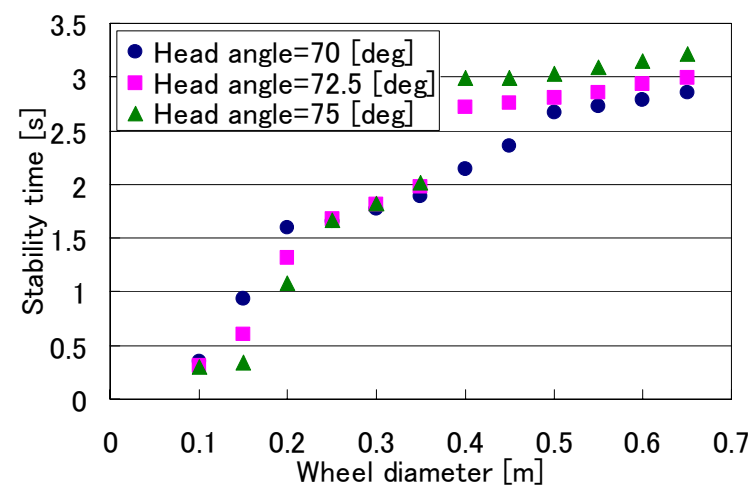

Fig. 8 Stability in different head angle 


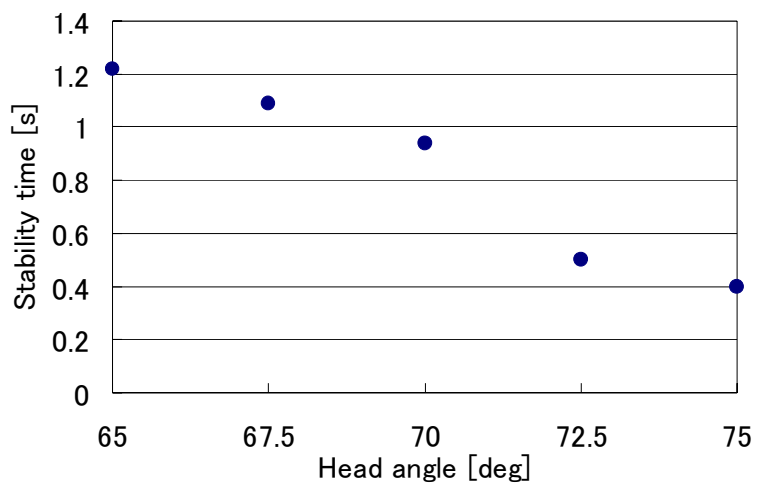

Fig. 9 Stability in different head angle for the small wheel bicycle

smaller wheel mass so that it has less stability in nature compared with the utility bicycle; the stability of the small wheel bicycle is highly sensitive to the variety of the trail length. Thus, it is found that the varying the head angle which decide the trail length has a possibility to greatly increase the stability of the small wheel bicycle. To examine more closely the influence of the head angle, Fig.9 shows the stability time at different head angles for the small wheel bicycle. Clearly, decreasing the head angle increases the stability time. In particular, it is shown that the small wheel bicycle with a 70 degree head angle has twice the stability time as that with a 75 degree head angle.

As for the stability time in Fig. 8, at the wheel diameter $0.4[\mathrm{~m}]$, the stability time has great difference in each head angle. In this case, the bicycle moves lateral translation for right direction, then it moves left direction near the stability limit before it crosses over. Therefore, it just increases the stability time.

From these results, it is found that the head angle is an important factor for the stability of the small wheel bicycles.

\section{Driving Experiment}

In the simulations, it has been found that the head angle is an influential parameter for the stability of the small wheel bicycle. In the experiments, the influence of the head angle is further examined by the riders. Although the simulation is performed assuming hand free riding, it is very dangerous to ride the small wheel bicycle by hand free so that the riders were allowed to use the handle here. Figure. 10 shows the experimental setup.

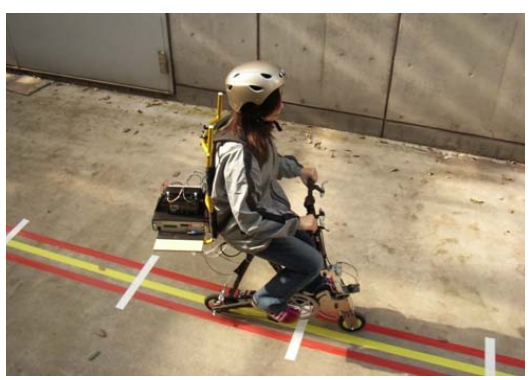

Fig. 10 Experimental setup

\subsection{Experimental Method}

The small wheel bicycle shown in Fig. 11 (a) is used in the experiment. The bicycle has 6-inch tires and a variable head angle mechanism. Figure. 11 (b) shows the variable head angle mechanism. The head angle is variable for the direction indicated by the arrow. The values of parameters are the same as those shown in Table 1. The subjects were five male 


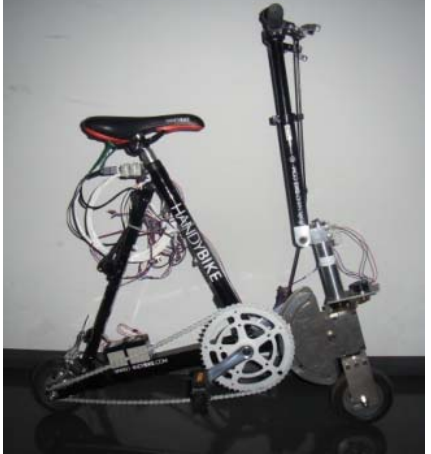

(a) Whole Body

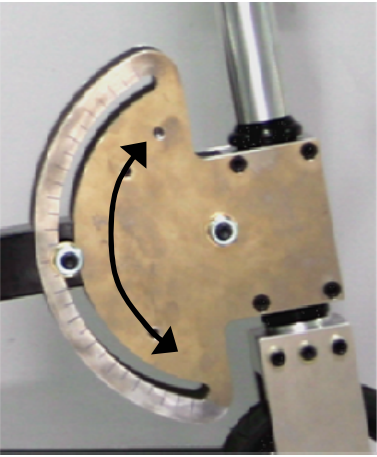

(b) Variable Head Angle Mechanism

Fig. 11 Experimental bicycle

riders (subjects weighting from 50 to $75[\mathrm{~kg}]$ ) who practiced riding the bicycle before the experiments. In the experiments, the subjects ride the bicycle through a $10[\mathrm{~m}]$ entrance length followed by a $10[\mathrm{~m}]$ measurement interval. The head angle is set to 70 and 75 degrees; each rider runs the experimental course twice for each head angle. The lateral translation, roll angle and steer angle were measured. The lateral translation was obtained by the image processing of a digital video captured from the front. The roll angle was calculated using the acceleration data obtained from a 3D inertial sensor mounted on the main frame. The steer angle was measured by a potentiometer on the steer axis. The questionnaire asked riders to choose condition which was more stable.

\subsection{Experimental Results}

Figures 12 and 13 show an example of the lateral translation and the roll angle measured by the experiment. It is shown that when the head angle is 70 degrees, the peaks of the lateral translation and roll angle are lower. The average lateral translation and roll angle for all subjects are shown in Figs. 14 and 15, the number of steering angle change are shown in Fig. 16. The average lateral translation is defined as the average of absolute value of the lateral translation. The average roll angle is defined as the average of absolute value of the roll angle from the upright position. The number of steering angle change is defined as the number of positive and negative change in steering angle rate. The average velocity of each of the subjects is about $2.5 \mathrm{~m} / \mathrm{s}$. In most of results, they show that the bicycle with a 70-degree head angle was more stable than that with a 75-degree for the subjects in terms of the lateral translation and roll angle. For the other results, in terms of the steering effort, the bicycle with a 70-degree head angle was superior to that with a 75-degree for the rest of the subjects. In Fig. 16, it is shown that the rest of the subjects had fewer steer angle changes for a head angle of 70 degrees. This means that the rider needs

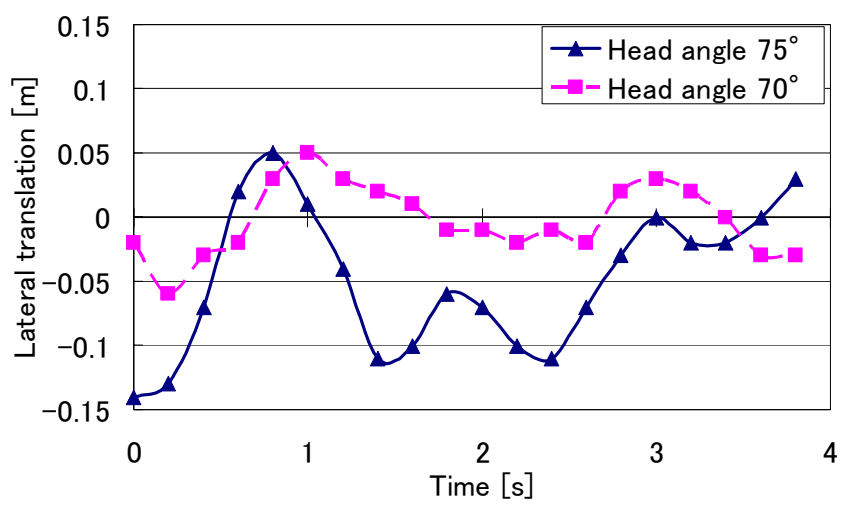

Fig. 12 Lateral translation by experiment 


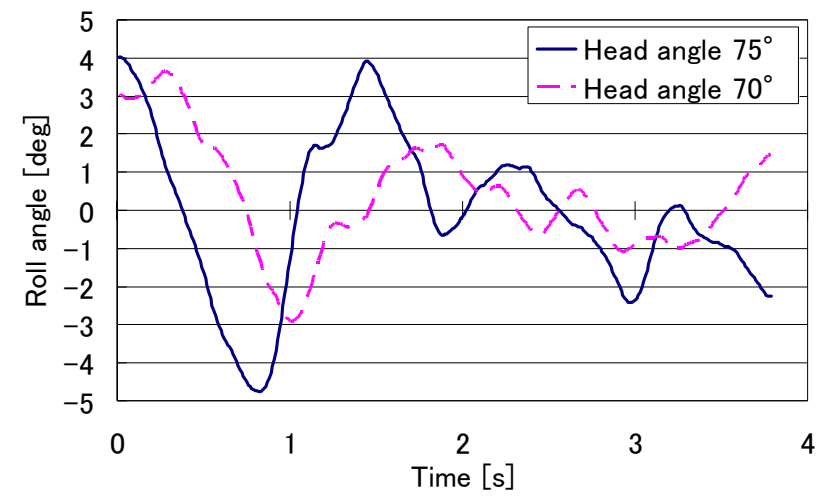

Fig. 13 Roll angle by experiment

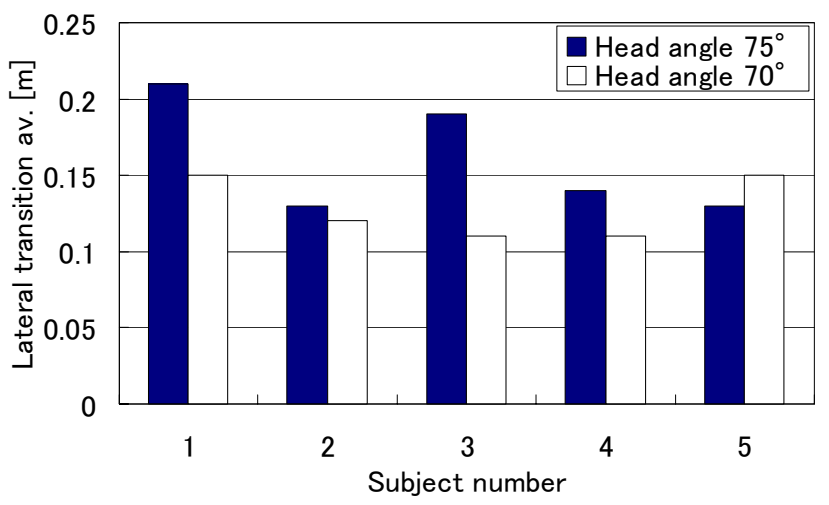

Fig. 14 Lateral transition average of each subject

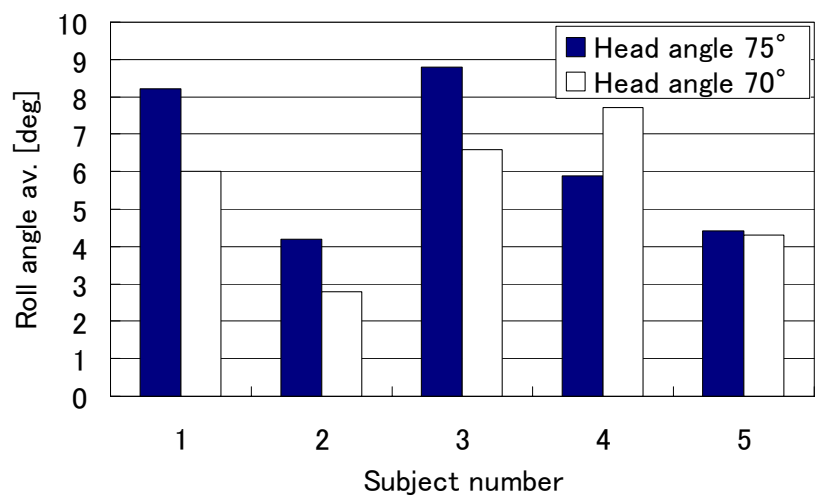

Fig. 15 Roll angle average of each subject

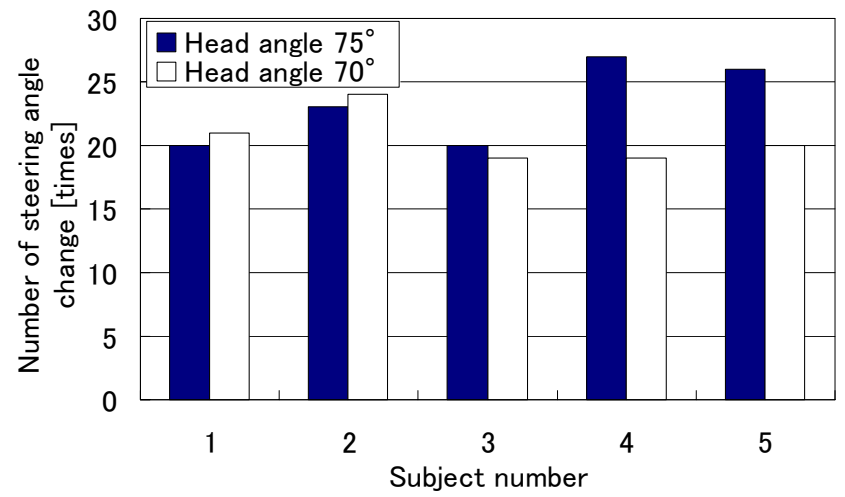

Fig. 16 Number of steer angle change of each subject 
less steering effort due to the self stabilization effect of the bicycle. Therefore, the measurement results show that the small wheel bicycle with a head angle of 70 degrees is more stable than that with a 75-degree.

The questionnaire results show that 4 out of 5 subjects chose a 70-degree head angle as the more stable small wheel bicycle, 1 out of 5 subjects answered neither and none of the subjects chose a 75-degree head angle.

From the driving experiments on the small wheel bicycle, it is confirmed that the small wheel bicycle with a head angle of 70 degrees is more stable than that with a 75-degree; that result correspond to the result obtained from the simulation.

\section{Evaluation of Parameter Effects}

Considering the improvement in the small bicycle stability, investigation on the effective parameter adjustment is necessary. In this chapter, the effect of parameters except for the head angle is proposed. As an example of increasing the stability of the small wheel bicycle, the numerical simulation is shown.

\subsection{Inertia Moment of the Wheel}

It is known that the gyro effect is one of the reasons for the bicycle stability. Then, the inertia moment of the wheel is one of the important parameter for the stabilization of the bicycle and should be investigated. This investigation can be used for the application to put electric motors in small wheels for the further development as a personal mobility vehicle. Assuming that the inertia moment of the wheel is varied by adding $2[\mathrm{~kg}]$ mass and made to be the circular disk, the standard wheel $A$ becomes the wheel $B$ which inertia moment is increased. Figure 17 shows the inertia moments of the wheel $A$ and the wheel $B$. The stability times of the bicycles with the wheel $A$ and the wheel $B$ are shown in Fig. 18. The initial velocity of the bicycle is $2.5[\mathrm{~m} / \mathrm{s}]$. Figure 18 shows that the stability time of the bicycle with the wheel diameter especially from 0.1 to $0.25[\mathrm{~m}]$ becomes longer even though the increase of the inertia moment of the wheels is small shown in Fig. 17. It means that the increasing the inertia moment has the large effect to the small wheel bicycle in particular. For example, the bicycle with the wheel diameter $0.15[\mathrm{~m}]$ has 2.5 times longer stability time when it increases the wheel inertia moment. Increasing the wheel mass to make its inertia moment larger leads to the increase of the normal force, this creates the trail effect and the front weight effect. By that effect, the produced self handling moment effectively improves the stability of the bicycle. Here we focus on the straight-line stability and upright stability. The stability concerning the maneuverability will be the future works.

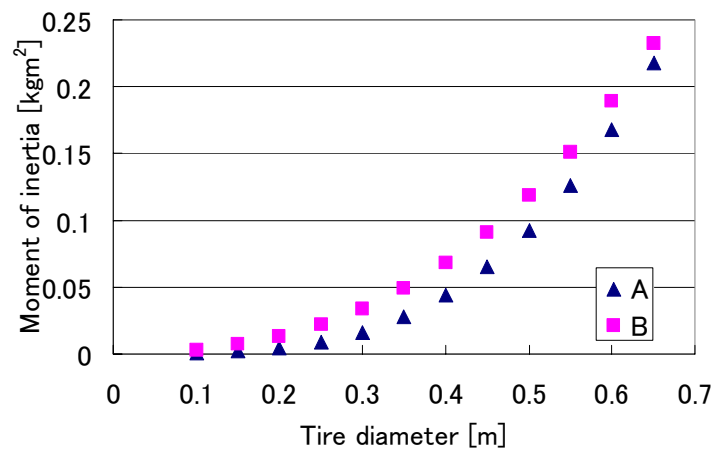

Fig. 17 Inertia moment of a wheel 


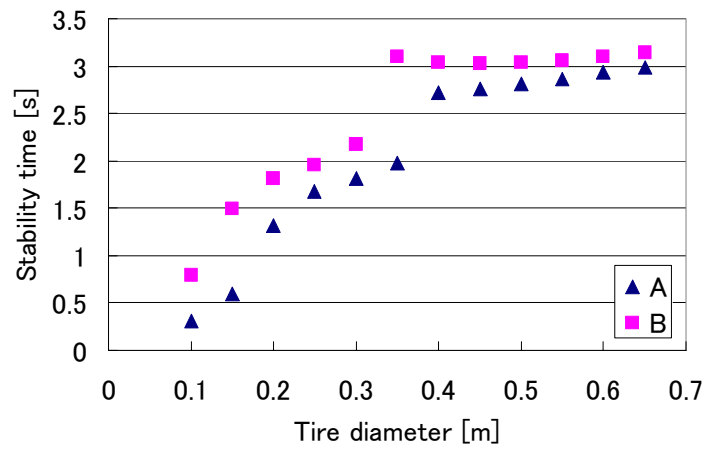

Fig. 18 Stability in different inertia moment of a wheel

\subsection{Numerical Simulation of the Small Wheel Bicycle Adjusted the Parameters.}

The stability of the small wheel bicycle with adjusting the parameters and without adjusting are compared in the numerical simulations. Table 2 shows the parameters of the improved small wheel bicycle. Figure 19 shows the simulation results of the small wheel bicycle with original parameters and improved. It is found that the small wheel bicycle with improved parameters has more stability than the original. Adapting the best combination of the parameters, the small wheel bicycle with tire diameter $0.15[\mathrm{~m}]$ improves its free hands running distance more than twice than of the original. This result indicates that there is a Table 2 Parameters of the improved small wheel bicycle possibility to increase the stability of the small wheel bicycle by the investigation method we proposed. It is also found that the small wheel bicycle has the unique potential to increase its stability effectively by adjusting the parameter.

\begin{tabular}{ll}
\hline Description & 6 inch bicycle (improved) \\
\hline Mass of main frame & $79 \mathrm{~kg}$ \\
Mass of front frame & $3.2 \mathrm{~kg}$ \\
Mass of tire & $2.62 \mathrm{~kg}$ \\
Tire diameter & $0.15 \mathrm{~m}$ \\
Wheelbase & $0.75 \mathrm{~m}$ \\
Offset & $0.035 \mathrm{~m}$ \\
Head angle & $70 \mathrm{deg}$ \\
\hline
\end{tabular}

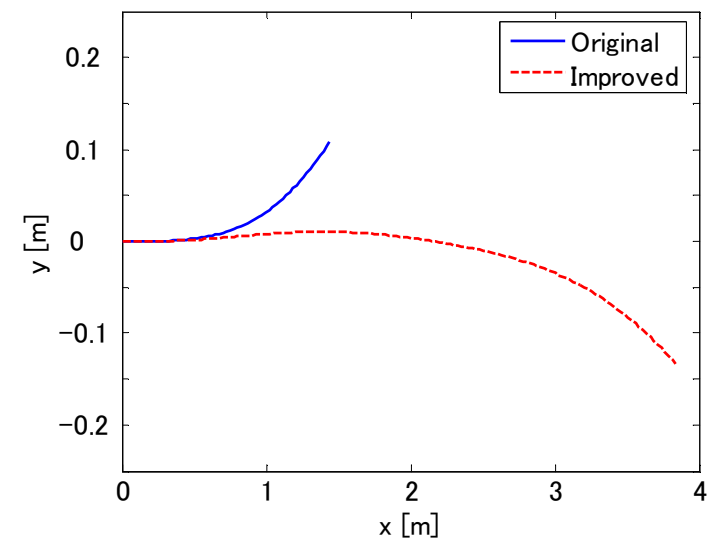

Fig. 19 Trajectories of bicycles (Original and improved) 


\section{Conclusion}

In this paper, the stability of the small wheel bicycles are investigated by using multibody dynamics and the driving experiments. The following conclusions are obtained.

(1) A multibody simulation model for a three-dimensional bicycle, which can consider the nonlinearity of the system with parameter change was proposed.

(2) The numerical simulations focusing on the wheel diameter showed that the stability of the bicycle sharply vary in the range of small wheel bicycles and indicate the effective parameters to improve their stability.

(3) Using the small wheel bicycle with variable head angle, the effect of the head angle was confirmed by the results of the driving experiments.

(4) It was found that increasing the inertia moment of the wheel of the small wheel bicycle improve its stability. The numerical simulation which applies the improved parameter indicated the possibility of increasing stability on the small wheel bicycle.

\section{References}

(1) Nguyen, H.G., Morrell, J., Mullens, K., Burmeister, A., Miles, S., Farrington, .N, Thomas, K., and Gage, DW., Segway Robotic Mobility Platform, Proceedings of Society of Photographic Instrumentation Engineers, No.5609 (2004)

(2) Kato, Y., Hosokawa, M., and Morita, M., Future Personal Mobility i-unit, Journal of Society of Automotive Engineers of Japan, Vol.60, No.2, (2006), pp.97-102

(3) Buntine, C., and Calstart, Clean Personal Mobility Services: A Transit-Enhancing Alternative to Private Automobile Travel, Transportation Research Board Annual Meeting CD-ROM, 03-4441, (2003)

(4) Brown, H.S., Vergragt, P., Green., K and Berchicci L., Learning for Sustainability Transition through Bounded Socio-technical Experiments in Personal Mobility, Technology Analysis \& Strategic Management, Vol. 15, No.3, (2003), pp.291-315

(5) Suda, Y., Iwasa, T., Miyamoto, T., Hirasawa, T. and Komine, H., Study of Intermodal Technology for Bicycle and Railway in Urban Transit, J-rail 2000, (1999), pp.129-132,

(6) Nakagawa, C., Nakano, K., Suda, Y., Kawarasaki, Y. and Kosaka, Y., Experiment on the Safety and Comfort of Personal Mobility Vehicles in Pedestrian Flows, Proceedings of JSAE First International Symposium on Future Active Safety Technology toward zero-traffic-accident, No. 20117349, (2011), pp.1-6

(7) TOYOTA MOTOR CORPORATION

http://www.toyota.co.jp/jp/tech/p_mobility/winglet/index.html

website,

(8) Sharp, R. S., The stability and control of motorcycles, Journal of Mechanical Engineering Science. Vol.13, (1971), pp.1305-1314

(9) Schwab, A.L, Meijaard, J.P and Papadopoulos, J.M, Benchmark Results on the Linearized Equation of Motion of an Uncontrolled Bicycle, KSME International Journal of Mechanical Science and Technology, Vol. 19, No.1, (2005), pp.292-304

(10) Kooijman, J. D. G., Schwab, A. L., and Meijaard, J. P, Experimental validation of a model of an uncontrolled bicycle, Multibody System Dynamics 19 (1-2) , (2008), pp.115-132

(11) Nakagawa, C., Suda, Y., Nakano K., and Takehara, S. , Stabilization of Two-Wheel Steering and Two-Wheel Driving Bicycle with Driving Forces, Journal of Mechanical Science and Technology, Vol.23, No.4, (2009), pp.980-986

(12) Nishimura, H., Zhu, S., Iwamatsu, S., Tajima, H., Modeling and Motion Control for Lane Change of Motorcycle, No.20-07 JSAE Symposium, The Latest Technologies of Motorcycles Dynamics and Safety, (2008), pp.10-14, (in Japanese)

(13) Japanese Industrial Standards committee, Cycles-Classification and essential characteristics, JIS D9111

(14) Japanese Industrial Standards committee, Method of stability test for bicycles, JIS D9203

(15) Ohashi, K., Kondo, T., Kobenno, Y., Washimi, K. and Hayashi, H., Bicycle traffic violations by high school students, Bicycle Technical Information No.70, (1996), pp.1-14 
(16) Japan bicycle promotion institute, Handbook of Bicycle for Actual Usage, (1971), pp. $27-28$

(17) Japan bicycle promotion institute, Handbook of Bicycle for Actual Usage, (1971), pp.239

(18) Society of Automotive Engineers of Japan, Automotive Engineering Handbook (Basic Theory), (2004), pp246

(19) Chen, C.K, Yang, C.K and Dao, T.S, Turning dynamics of two-wheeled vehicles, Proceedings of Fourth Asian Conference on Multibody Dynamics, (2004), pp.390-398

(20) Marumo, Y. and Nagai, M,, Steering control of motorcycles using steer-by-wire system, Vehicle System Dynamics, Vol.45, No.5, (2007), pp.445-458

(21) Cossalter, V. and Sadauckas, J., Elaboration and quantitative assessment of manoeuvrability for motorcycle lane change, Vehicle System Dynamics, Vol.44, No.12, (2006), pp.903-920 